\title{
Estudiantes Del Título Superior De Música De Viento Madera. Relación Entre Los Niveles Motivacionales Y El Optimismo Disposicional
}

\author{
Francisco Javier Zarza-Alzugaray, Dr. \\ Universidad Complutense de Madrid, Spain
}

Doi: 10.19044/esj.2018.v14n11p1 URL:http://dx.doi.org/10.19044/esj.2018.v14n11p1

\begin{abstract}
The motivation and the dispositional optimism are two constructs that in the population of musicians in training have not yet been taken into account in order to their scientific study. However, we present an approach to the problem, adapting it to the musical contexts, based on the motivational model proposed by Manassero and Vázquez (1998); the motivational factors are the interest, effort, perfectionism, the specific demands of the task and the exams. At the same time, taking as valid the approach to the model of optimism from Carver, Scheier and Segerstrom (2010), in which the optimistic tendency is defined as a belief that each person has in his life towards the experience good experiences, significant relationships have been found between the different motivational factors and optimism; at the same time and with regression techniques it has been possible to explain $29.4 \%$ of the variance of the general factor of optimism with only the significant presence in the model of the specific factor of the task. In this sense, more research is needed to increase the knowledge of both constructs for the improvement of pedagogical practice in higher musical training centers.
\end{abstract}

Keywords: Music education, music students, dispositional optimism, achieve motivation

\section{Resumen}

La motivación y el optimismo disposicional son dos constructos que en la población de músicos en formación no han sido tenidos en cuenta de cara a su estudio científico. Sin embargo, presentamos una aproximación al problema, adaptándolo a los contextos musicales, basándonos en el modelo motivacional propuesto por Manassero y Vázquez (1998); se describen como factores clave motivacionales el interés, el esfuerzo, el perfeccionismo, las demandas específicas de la tarea y los exámenes. A su vez, tomando como válida la aproximación al modelo de optimismo de Carver, Scheier y 
Segerstrom (2010), en el que se define la tendencia optimista como creencia que cada persona tiene de que en su vida experimentará buenas experiencias, se han encontrado relaciones significativas entre los distintos factores motivacionales y el optimismo; del mismo modo que a partir de técnicas de regresión se ha conseguido explicar el $29.4 \%$ de la varianza del factor general de optimismo con únicamente la presencia significativa en el modelo del factor específico de la tarea. En este sentido se hace necesaria más investigación que permita ahondar más en el conocimiento de ambos constructos para la mejora de la práctica pedagógica en los centros formativos musicales superiores.

Palabras-clave: Educación musical, estudiantes de música, optimismo disposicional, motivación de logro

\section{Introducción}

El concepto de motivación ha experimentado una larga trayectoria en la investigación desde que en 1925 Tolman lo introdujese como constructo a tener en cuenta debido a su potencia explicativa y predictiva de la conducta en contextos muy diferentes y diversos (Manassero y Vázquez, 1998). A pesar de las teorías existentes, para Fenouillet la motivación de logro responde en última instancia a la obtención de cierta satisfacción psicológica por el logro obtenido y en donde al final lo que se persigue es la obtención de placer personal (Fenouillet, 2009, 2012).

Diversos investigadores sugieren que la motivación, en términos generales, no puede explicarse a partir de un solo referente teórico, sino que de forma segura, está configurada de diferentes tipos de motivación relativos a distintos niveles y ámbitos de actuación (Elias, Mustafa, Roslan y Noah, 2011). Los objetivos que las personas adoptan en un entorno de aprendizaje tiene importantes implicaciones en un ámbito muy amplio en los comportamientos académicos (Meece, Glienke y Burg, 2006) y existen pruebas en estudios universitarios que sugieren que las actuaciones enfocadas a la obtención de una meta están relacionadas positivamente con los resultados futuros (Harackiewicz, Barron, Pintrich, Elliot y Thrash, 2002).

En este sentido, se entiende la motivación de logro como "la tendencia a conseguir una buena ejecución en situaciones que implican competición con una norma o un estándar de excelencia, siendo la ejecución evaluada como éxito o fracaso, por el propio sujeto o por otros" (Garrido, 1986, p. 138); o también y más breve como "la motivación que impulsa la ejecución en las denominadas tareas de logro" (Manassero y Vázquez, 1998, p. 334).

En el ámbito de la motivación de logro toma especial importancia el estilo atributivo de los eventos negativos, bien como antecesoras de la conducta para conseguir un logro u objetivo, bien como consecuencia de la conducta encaminada a la obtención de un logro determinado (Kelley y 
Michella, 1980). También es de recalcar que otra de las facetas que son motivo de estudio de la motivación de logro deben ser los aspectos relacionados con el yo, entendido éste como la sede de las creencias y de las actitudes, así como el soporte de las antedichas adscripciones causales (Manassero y Vázquez, 1998); en donde van a tener cabida constructos autorregulatorios como el autoconcepto, autoestima, y directamente relacionado con esta investigación la autoeficacia.

Una aproximación atribucional al comportamiento de los estudiantes, puede dirigir las expectativas de la consecución de un éxito y puede llegar a influir en sentimientos como el orgullo, la culpa, la vergüenza o la autoestima (Weiner, 2000). Más recientemente y coligiendo la motivación de logro con el optimismo y el pesimismo, está reportado que un estilo atribucional de corte optimista se muestra como un predictor potente en la motivación hacia la consecución de una tarea determinada, mientras que un estilo atribucional de corte pesimista, presenta unas actuaciones menores o el abandono en el intento del logro de la misma (Gordon, 2008).

El optimismo se define como un constructo psicológico que plantea diferencias individuales en lo relativo a las expectativas futuras. Desde un punto de vista científico el optimismo disposicional, relaciona las expectativas positivas de acontecimientos futuros con la confianza de que éstos serán logrados (Carver, Reynolds y Scheier, 1994; Carver y Scheier, 2000; Orejudo y Teruel, 2009; Peterson, 2000).

Se puede decir por lo tanto que el optimismo es un constructo que relaciona la consecución de una meta establecida, con las conductas y pensamientos encaminados hacia la consecución de la misma. Sin embargo, la percepción del posible logro de la meta mediante una serie de actuaciones, está condicionada, al igual que las expectativas, por los procesos de aprendizaje que se han tenido hasta ese momento encontrando diferencias individuales en estos procesos (Zarza, 2014).

El objetivo principal de este estudio es indagar en las relaciones que mantengan la motivación de logro y el optimismo disposicional en los estudiantes superiores de música españoles, concretamente de instrumentos de viento madera.

\section{Método}

\section{Participantes}

La muestra está compuesta por 130 estudiantes (58 hombres y 72 mujeres) del Título Superior de Música pertenecientes a 5 centros de formación superior españoles.

Todos los estudiantes son intérpretes de instrumentos de viento madera (42 clarinete, 37 flauta, 30 saxofón, 9 fagot, 9 oboe, 1 traverso barroco y 1 
flauta de pico). Presentan edades comprendidas entre los 16 y los 45 años con una edad media de 22.12 (D.T. $=3.25$ ).

\section{Procedimiento}

Los datos fueron recogidos en los cinco centros superiores que participaron en la investigación. Para ello un miembro del equipo de investigación se desplazó a los distintos centros, previo acuerdo con la dirección de los mismos, para tratar de realizar un mejor y más eficiente acopio de cuestionarios.

\section{Instrumentos y análisis de datos}

Se ha empleado la escala de motivación de logro en contextos musicales (Zarza, 2014) para el estudio de la motivación de logro. El cuestionario consta de 26 preguntas que son evaluadas en una escala Likert de 9 puntos; el cómputo general de la misma arroja un estadístico de fiabilidad $\alpha$ de Cronbach de 0.775. La versión confirmatoria de 18 ítems ( $\alpha$ de Cronbach de 0.774 ) presenta 5 subescalas diferenciadas: interés (4 ítems), esfuerzo (2 ítems), perfeccionismo ( 2 ítems), tarea (8 ítems) y examen (2 ítems).

Respecto a la escala de optimismo, se ha empleado la versión española de Otero et al. (1998) del LOT-R, Life Orientation Test, de Scheier, Carver y Bridges (1994). Es un cuestionario usado para evaluar el optimismo y el pesimismo disposicional y constituye un instrumento válido y muy utilizado por la comunidad científica para valorar el papel del optimismo en diferentes áreas de conocimiento (Ruthig, Raymond, Hall y Hladkyj, 2004) como la salud, el deporte o valoraciones académicas (Chang, 1998; Hellandsig, 1998; Stoecker, 1999); en esta investigación se ha empleado la adaptación al castellano que hicieron Otero et al. en 1998. Es un instrumento que está constituido por diez enunciados (Likert de 5 puntos), en donde tres ítems miden los niveles de optimismo disposicional y tres el pesimismo disposicional. Tras la inversión y posterior sumatorio de las puntuaciones se obtiene una valoración de optimismo disposicional general en donde a mayores puntuaciones mayor optimismo disposicional.

Relativo al estudio estadístico de datos, se han llevado a cabo análisis descriptivos y correlacionales con los programas informáticos SPSS en su versión 19.0.

\section{Resultados}

Respecto a los resultados de los distintos factores motivacionales se observa cómo todos ellos, excepto el de perfeccionismo, están por encima de la media teórica del instrumento (Tabla 1). Así, observamos además que estas diferencias con respecto a la media son significativamente superiores para el interés $(\mathrm{t}=33.025 ; \mathrm{p}=.000)$, para el esfuerzo $(\mathrm{t}=17.588 ; \mathrm{p}=.000)$, para el 
factor tarea $(\mathrm{t}=9.833 ; \mathrm{p}=.000)$ y para el factor exámenes $(\mathrm{t}=3.351 ; \mathrm{p}=.001)$ siendo significativamente inferior también para el factor perfeccionismo $(\mathrm{t}=$ 7.144; $\mathrm{p}=.000)$.

Tabla1. Estadísticos descriptivos.

\begin{tabular}{|c|c|c|c|c|c|c|}
\hline & $\mathrm{N}$ & Mínimo & Máximo & Media & $\begin{array}{c}\text { Media } \\
\text { Teórica }\end{array}$ & Desv. típ. \\
\hline Interés & 130 & 8.00 & 36.00 & 32.1215 & 20 & 4.18483 \\
Esfuerzo & 130 & 2.00 & 18.00 & 14.4862 & 10 & 2.90836 \\
Perfeccionismo & 130 & 2.00 & 18.00 & 7.3231 & 10 & 4.27217 \\
Tarea & 130 & 10.00 & 64.00 & 43.0248 & 40 & 10.29254 \\
Exámenes & 130 & 5.00 & 18.00 & 12.1077 & 10 & 2.44393 \\
N válido (según lista) & 140 & & & & & \\
\hline
\end{tabular}

Una mirada más detallada a los resultados de distribución muestral pone de manifiesto que el $15.4 \%$ de los estudiantes presentan una puntuación inferior en más de una desviación típica a la media muestral en el factor interés, este hecho alcanza un porcentaje del $9.2 \%$ en el factor esfuerzo. Con respecto al factor de perfeccionismo el $20 \%$ de los estudiantes presenta una media alejada por la parte negativa en más de una desviación típica y en lo que respecta al factor tarea se sitúa este porcentaje en el 15.4\% siendo el del factor exámenes del $12.6 \%$.

En cuanto al análisis correlacional de los distintos factores en los que podemos dividir la motivación, se comprueba que el interés correlaciona de manera significativa y directamente proporcional con el esfuerzo $(r=.622 ; \mathrm{p}$ $=.000)$, con la tarea $(\mathrm{r}=.300 ; \mathrm{p}=.001)$ y con el factor exámenes $(\mathrm{r}=.276 ; \mathrm{p}$ $=.001)$; mientras que lo hace de manera inversamente proporcional con el factor perfeccionismo $(\mathrm{r}=-.267 ; \mathrm{p}=.002)($ Tabla 2$)$.

Tabla 2. Correlaciones.

\begin{tabular}{|c|c|c|c|c|c|}
\hline & Interés & Esfuerzo & Perfeccionismo & Tarea & Exámenes \\
\hline Interés & 1 & $.622^{* *}$ & $-.267^{* *}$ & $.300^{* *}$ & $.276^{* *}$ \\
\hline Esfuerzo & $.622^{* *}$ & 1 & $-.192^{*}$ & $.188^{*}$ & $.195^{*}$ \\
\hline Perfeccionismo & $-.267^{* *}$ & $-.192^{*}$ & 1 & & \\
\hline Tarea & $.300^{* *}$ & $.188^{*}$ & & 1 & $.491^{* *}$ \\
\hline Exámenes & $.276^{* *}$ & $.195^{*}$ & & $.491^{* *}$ & 1 \\
\hline
\end{tabular}

Respecto al constructo del optimismo, se ha obtenido una puntuación media igual a 20.084 puntos $($ D.T. $=4.38)$ con un rango de puntuaciones desde 6 puntos (la mínima posible) hasta 30 puntos (la máxima posible). Además, se ha comprobado que la media obtenida es significativamente superior $(\mathrm{t}=$ $5.425 ; \mathrm{p}=.000)$ a la media teórica del instrumento (18 puntos). En este sentido vemos cómo en torno al $16.2 \%$ de los estudiantes presentan una puntuación media inferior en más de una desviación típica a la media (Figura 1). 


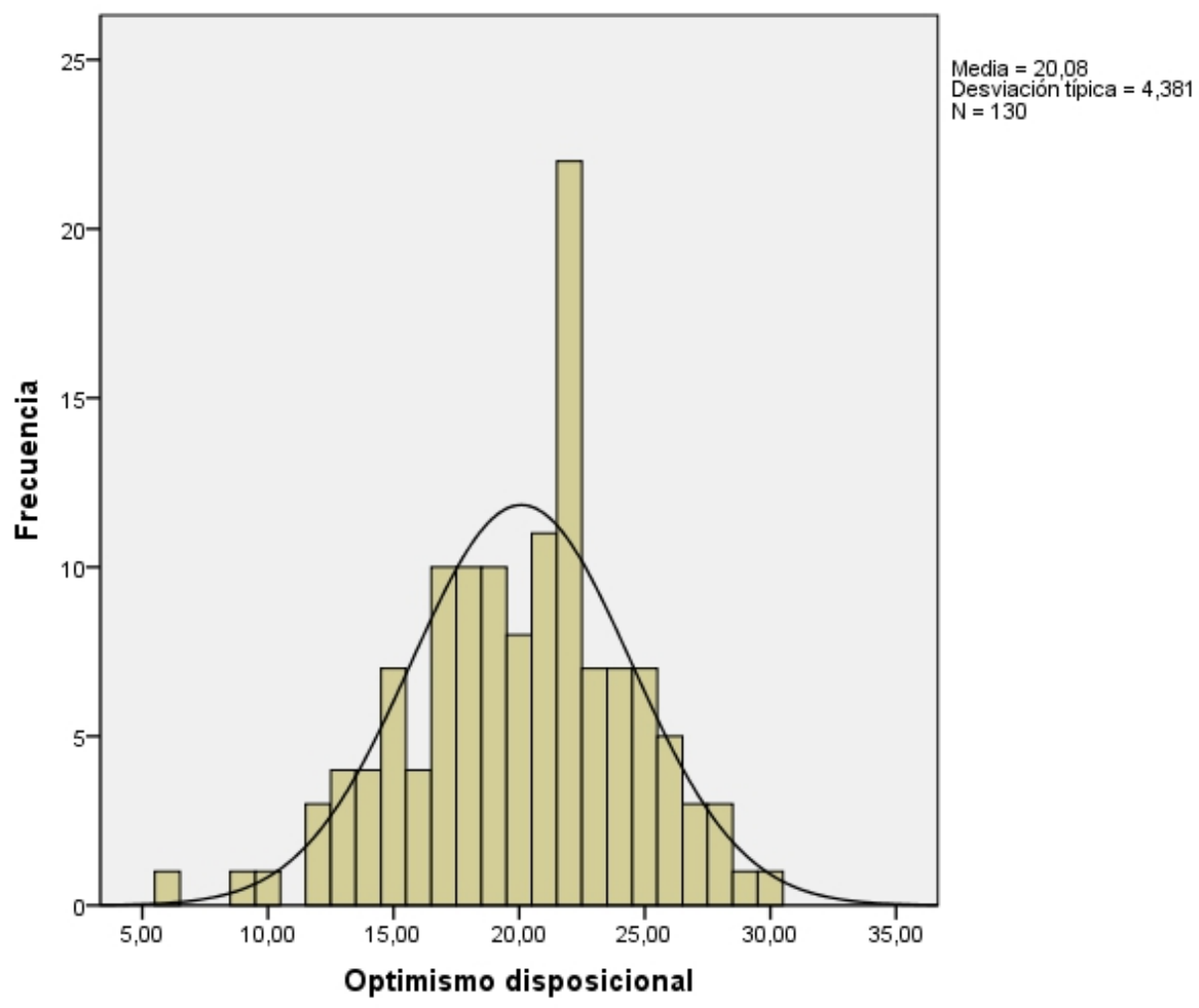

Figura 1. Distribución de la muestra.

De manera correlacional, se observa cómo el optimismo correlaciona significativamente y de manera directamente proporcional con los factores de interés $(\mathrm{r}=.234 ; \mathrm{p}=.007)$, con el de tarea $(\mathrm{r}=.545 ; \mathrm{p}=.000)$ y con el de exámenes $(\mathrm{r}=.367 ; \mathrm{p}=.000)$ mientras que no presenta asociación correlacional con los factores de esfuerzo $(\mathrm{r}=.106 ; \mathrm{p}=.231) \mathrm{ni}$ perfeccionismo $(\mathrm{r}=.130 ; \mathrm{p}=.140)$.

Tabla 3. Correlaciones. Motivación - Optimismo disposicional.

\begin{tabular}{|c|c|c|c|c|c|}
\hline & Interés & Esfuerzo & Perfeccionismo & Tarea & Exámenes \\
\hline Optimismo disposicional & $.234^{* *}$ & .106 & .130 & $.545^{* *}$ & $.367^{* *}$ \\
\hline
\end{tabular}

En este punto de estudio de las relaciones entre ambos constructos, se considera de interés estudiar cómo los factores motivacionales pueden estar influyendo en las diferencias de niveles de optimismo entre los estudiantes. De esta forma se plantea una regresión con el método enter que dará cuenta del aporte significativo de cada uno de los componentes motivacionales en la varianza del factor general de optimismo disposicional.

Se llega de esta manera a un modelo con capacidad explicativa significativa $\left(\mathrm{F}=11.751 ; \mathrm{p}=.000 ; \mathrm{D}-\mathrm{W}=1.898 ; \mathrm{R}^{2}=.294\right)$ que es capaz de explicar el $29.4 \%$ de la varianza total del optimismo disposicional. 
La varianza explicada de la variable dependiente, como puede apreciarse en la Tabla 4, viene explicada exclusivamente de manera significativa por la acción del componente motivacional específico de la tarea $(\mathrm{t}=5.156 ; \mathrm{p}=.000 ; \beta=.452)$; mientras que los componentes de interés $(\mathrm{t}=$ $1.282 ; \mathrm{p}=.202 ; \beta=.130)$, esfuerzo $(\mathrm{t}=-.702 ; \mathrm{p}=.484 ; \beta=-.066)$, perfeccionismo $(\mathrm{t}=1.003 ; \mathrm{p}=.318 ; \beta=.080)$ y exámenes $(\mathrm{t}=1.309 ; \mathrm{p}=.193$; $\beta=.113$ ) no presentan carga significativa en el modelo.

Tabla 4. Regresión. Método Enter. Variable dept.: Optimismo disposicional.

\begin{tabular}{|c|c|c|c|c|c|c|c|c|}
\hline & \multirow{2}{*}{$\begin{array}{c}\text { Coeficientes } \\
\text { tipificados } \\
\text { Beta }\end{array}$} & \multirow{2}{*}{$\mathrm{t}$} & \multirow{2}{*}{ Sig. } & \multicolumn{2}{|c|}{$\begin{array}{c}\text { Estadísticos de } \\
\text { colinealidad }\end{array}$} & \multirow{2}{*}{$\mathrm{F}$} & \multirow{2}{*}{ Sig } & \multirow{2}{*}{$\begin{array}{l}\text { Durbin- } \\
\text { Watson }\end{array}$} \\
\hline & & & & Tolerancia & FIV & & & \\
\hline Interés & .130 & 1.282 & .202 & .535 & 1.869 & \multirow{5}{*}{11.751} & \multirow{5}{*}{.000} & \multirow{5}{*}{1.898} \\
\hline Esfuerzo & -.066 & -.702 & 484 & .612 & 1.634 & & & \\
\hline Perfeccionismo & .080 & 1.003 & .318 & .866 & 1.154 & & & \\
\hline Tarea & .452 & 5.156 & .000 & .711 & 1.406 & & & \\
\hline Exámenes & .113 & 1.309 & \begin{tabular}{|l|}
.193 \\
\end{tabular} & .730 & 1.369 & & & \\
\hline
\end{tabular}

\section{Conclusión}

Encuadrándose en un el contexto formativo musical español, se ha podido poner de manifiesto cómo los constructos del optimismo y la motivación de logro presentan una fuerte relación. Además, profundizando más en el constructo motivacional y estudiando sus factores diferenciales (interés, esfuerzo, perfeccionismo, tarea y exámenes) presentados por Manassero y Vázquez (1998) en relación a la capacidad explicativa y posible funcionamiento correlacional con el optimismo, se ha visto que aparecían ciertas diferencias. Así el papel del perfeccionismo y el esfuerzo no ha quedado claro de manera correlacional y se hace necesaria, por lo tanto, mayor investigación. Sin embargo, el factor motivacional asociado a las demandas específicas de la tarea ha presentado una fuerte asociación con el optimismo disposicional.

Estos resultados se pueden enlazar con investigaciones paralelas en las que se estudian las relaciones entre características optimistas y motivacionales (Falcón, Orejudo, Fernández-Turrado y Zarza, 2016). En este sentido, también se hace necesario más estudio con diversas técnicas estadísticas que puedan esclarecer el posible papel mediador que puedan tener los demás componentes motivacionales de cara a explicar las diferencias en los niveles de optimismo y de motivación de la población de estudiantes de música como se puede ver en Zarza, Casanova y Orejudo (2016); así como la relación de estos constructos con otros que en la literatura científica se relacionan como puedan ser la ansiedad, la sensibilidad al premio o al castigo o la autoeficacia (Torrubia, Ávila, Moltó y Caseras, 2001). 


\section{References:}

1. Carver, C. S., Reynolds, S. L., \& Scheier, M. F. (1994). The possible selves of optimists and pessimists. Journal of Research in Personality, 28, 133-141.

2. Carver, C. S. \& Scheier, M. F. (2000). Perspectives on personality (4th ed.). Needham Heights, MA: Allyn \& Bacon.

3. Carver, C. S., Scheier, M. F., \& Segerstrom, S. C. (2010). Optimism. Clinical Psychology Review, 30(7), 879-889. doi:10.1016/j.cpr.2010.01.006

4. Chang, E. C. (1998). Does dispositional optimism moderate the relation between perceived stress and psychological well-being? A preliminary investigation. Personality and Individual Differences, 25(2), 233-240. doi:10.1016/S0191-8869(98)00028-2

5. Elias, H., Mustafa, S. M. S., Roslan, S., \& Noah, S. M. (2011). Motivational predictors of academic performance in end year examination. Procedia - Social and Behavioral Sciences, 29, 11791188.

6. Falcón, C., Orejudo, S., Fernández-Turrado, T., \& Zarza, J. (2016). Emergencia y evolución de las expectativas optimistas en niños de educación primaria. Anales de Psicología, 32(2), 492-500. doi:10.6018/analesps.32.2.239351

7. Fenouillet, F. (2009). Vers une intégration des conceptions théoriques de la motivation. Habilitation à diriger des recherches. Paris: Université Paris Ouest la Défense.

8. Fenouillet, F. (2012). Les conceptions hédoniques de la motivation. Pratiques Psychologiques, 18, 121-131.

9. Garrido, I. (1986). La motivación escolar: determinantes sociológicos y psicológicos del rendimiento. In J. Mayor (Ed.), Sociología y Psicología de la Educación (pp. 122-151). Madrid, Spain: Anaya.

10. Gordon, R. A. (2008). Attributional style and athletic performance: Strategic optimism and defensive pessimism. Psychology of Sport and Exercise, 9, 336-350.

11. Harackiewicz, J. M., Barron, K. E., Pintrich, P. R., Elliot, A. J., \& Thrash, T. M. (2002). Revision of achievement goal theory: Necessary and illuminating. Journal of Educational Psychology, 94, 638-645.

12. Hellandsig, E. T. (1998). Motivational predictors of high performance and discontinuation in different types of sports among talented teenage athletes. International Journal of Sport Psychology, 29, 27-44.

13. Kelley, H. H. \& Michela, J. L. (1980). Attribution theory and research. Annual Review of Psychology, 31, 457-501.

14. Manassero, M. A. \& Vázquez, A. (1998). Validación de una escala de motivación de logro. Psicothema, 10(2), 333-351. 
15. Meece, J. L., Glienke, B. B., \& Burg, S. (2006). Gender and motivation. Journal of school Psychology, 44, 351-373.

16. Orejudo, S. \& Teruel, M. P. (2009). Una mirada evolutiva al optimismo en la edad escolar. Algunas reflexiones para padres, educadores e investigadores. Revista Interuniversitaria de Formación del Profesorado, 66(23,3), 129-158.

17. Otero, J. M., Luengo, A., Romero, E. Gómez, J. A., \& Castro, C. (1998). Psicología de personalidad. Manual de prácticas. Barcelona: Ariel Practicum.

18. Peterson, C. (2000). The future of optimism. American Psychologist, 55(1), 44-55. doi:10.1037/0003-066X.55.1.44

19. Ruthig, J., Raymond, P., Hall, N., \& Hladkyj, S. (2004). Optimism and attributional retraining: longitudinal effects on academic achievement, test anxiety, and voluntary course withdrawal in college students. Journal of Applied Social Psychology, 34(4), 709-730.

20. Scheier, M. F., Carver, C. S., \& Bridges, M. W. (1994). Distinguishing optimism from neuroticism (and trait anxiety, self-mastery, and selfesteem): A reevaluation of the Life Orientation Test. Journal of Personality and Social Psychology, 51, 1257-1264.

21. Stoecker, J. A. (1999). Optimism and grade expectancies. Psychological Reports, 84, 873-879.

22. Torrubia, R., Ávila, C., Moltó, J., \& Caseras, X. (2001). The sensitivity to punishment and sensitivity to reward questionnaire (SPSRQ) as a measure of Gray's anxiety and impulsivity dimensions. Personality and Individual Differences, 31(6), 837-862. doi:10.1016/S01918869(00)00183-5

23. Weiner, B. (2000). Interpersonal and intrapersonal theories of motivation from an attributional perspective. Educational Psychology Review, 12, 1-14.

24. Zarza, F. J. (2014). Variables psicológicas y pedagógicas como predictoras de la ansiedad escénica en estudiantes de Grado Superior de Música de España. Tesis doctoral. Zaragoza, Spain: Universidad de Zaragoza.

25. Zarza, F. J., Casanova, O., \& Orejudo, S. (2016). Ansiedad escénica y constructos psicológicos relacionados. Estudiantes de cinco conservatorios superiores de música españoles. RIEM. Revista Internacional de Educación Musical, 4, 13-24. doi:10.12967/RIEM2016-4-p013-024 